\title{
Meningitis neumocóccica por serotipo 19 A, de curso fatal. ¿Un caso prevenible?
}

\author{
M. Carolina Rivacoba, José Cofré y M. Elena Santolaya
}

\section{Fatal pneumococcal meningitis due to serotype 19 A. A preventable case?}

Invasive pneumococcal disease (IPD) remains as an important cause of morbidity in the world and in our country, while in Chile the incidence has decreased after the incorporation of the 10 valent pneumococcal conjugate vaccine, in the routine infant inmunization schedule (EPI). One of the expected effects of the program after vaccination with 10 -valent pneumococcal vaccine is the likely replacement serotype phenomenon that means the presence of ENI caused by serotypes not included in the vaccine. In this context, we present the case of a child with pneumococcal meningitis caused by serotype 19 A of fatal course. The occurrence of ENI in a later stage of pneumococcal vaccine incorporation in Chile reinforces the importance of active surveillance, in order to know in detail the impact of vaccination, distribution of circulating serotypes and their correlation with the different clinical disease and their severity.

Key words: Invasive pneumococcal disease, serotype 19 A, serotypes replacement.

Palabras clave: Enfermedad neumocóccica invasora, serotipo 19 A, reemplazo de serotipos.

\section{Introducción}

$\mathrm{S}$ e ha definido como enfermedad neumocóccica invasora (ENI) a la detección de Streptococcus pneumoniae en un sitio estéril tal como sangre, líquido cefalorraquídeo (LCR), líquido pleural, articular, peritoneal o pericárdico. Esta pesquisa puede ser por cultivo, por detección de antígenos de superficie de su cápsula y más recientemente mediante amplificación genética del ADN bacteriano. La ENI es una enfermedad grave, ligada a hospitalización y muerte. Según datos aportados por la Organización Mundial de la Salud, 1,6 millones de niños menores de cinco años presentan un episodio de ENI y anualmente se producen entre 12.000 a 28.000 muertes $^{1}$.

Existen más de 90 serotipos de S. pneumoniae, los que están determinados por el polisacárido de su cápsula, reconociéndose algunos de ellos como más agresivos y causantes de infecciones invasoras. Las infecciones por S. pneumoniae representan la primera causa de muerte infantil por enfermedades inmunoprevenibles ${ }^{2}$.

Se presenta el caso clínico de un pre-escolar con meningitis causada por S. pneumoniae serotipo 19 A y luego, una discusión respecto a características propias de este agente, así como el panorama sero-epidemiológico obtenido mediante la vigilancia nacional de laboratorio de las ENI. Finalmente, se enfatiza la importancia de estudiar una probable inmunodeficiencia en relación a casos de ENI en niños vacunados.

\section{Caso clínico}

Pre-escolar de 2 años 2 meses, sexo masculino, sin antecedentes mórbidos de importancia. Tenía el esquema de vacunación, según el Programa Nacional de Inmunizaciones (PNI) chileno al día, incluyendo tres dosis de vacuna antineumocóccica 10-valente, a los 2-4 y 12 meses de edad. Había recibido además las vacunas extra-PNI anti-varicela y hepatitis A. Asistía al jardín infantil hacía un año.

El cuadro se inició $72 \mathrm{~h}$ previas a su consulta, caracterizado por compromiso del estado general, asociado a fiebre y vómitos explosivos en dos oportunidades.

Persistió febril hasta $38,2^{\circ}$, acentuándose el compromiso del estado general, por lo que consultó en Servicio de Urgencia, donde se constató un paciente comprometido de conciencia, Glasgow 9, febril, con aumento de la presión diferencial sisto-diastólica (130/60), llene capilar límite y con signos meníngeos. Se diagnosticó un shock séptico y una probable meningitis bacteriana aguda. Se inició manejo del shock con volumen i.v. y asistencia ventilatoria. Por el compromiso de conciencia se realizó una TAC de cerebro que no evidenció signos de hipertensión endocraneal y a continuación se le efectuó una punción lumbar que dio salida a LCR turbio. El análisis citoquímico fue: hipoglucorraquia (glucosa $<10 \mathrm{mg} / \mathrm{dl}$ ), proteinorraquia $515 \mathrm{mg} / \mathrm{dl}$, eritrocitos $10 / \mathrm{mm}^{3}$, leucocitos $80 / \mathrm{mm}^{3}(60 \%$ polimorfonucleares y $40 \%$ mononucleares). En sus exámenes generales destacaban un recuento hema-
Facultad de Medicina, Universidad de Chile. Departamento de Pediatría y Cirugía Infantil, Campus Oriente (MES). Programa de formación de Infectología pediátrica (MCR). Hospital de niños Dr. Luis Calvo Mackenna. Unidad de Infectología (JC).

Los autores declaran no tener conflictos de interés en este tema. Recibido: 29 de julio de 2015 Aceptado: 22 de diciembre de 2015

Correspondencia a: M. Carolina Rivacoba R. carorivacoba@gmail.com 
Figura 1. Tinción de Gram directa de líquido cefalorraquídeo de ingreso, visualizado bajo MO 60x. Se observan cocáceasgrampositivas en abundancia y la virtual ausencia de leucocitos.

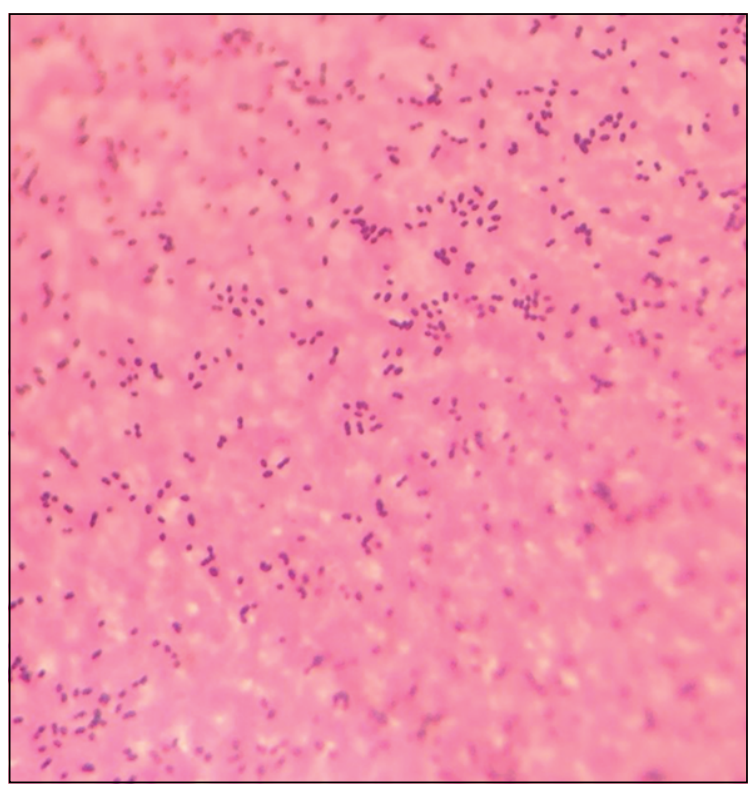

Tabla 1. Evolución de recuento de leucocitos y valores de proteína C reactiva en un preescolar con meningitis neumocóccica

\begin{tabular}{ccccc}
\hline Examen & Hto(\%)/Hb (g/d) & Plaquetas $/ \mathbf{m m}^{3}$ & $\begin{array}{c}\text { Leucocitos } / \mathbf{m m}^{3} / \% \\
\text { segmentados }\end{array}$ & PCR (mg/L) \\
Día 1 & $35,4 / 12,2$ & 224.000 & $5.800 / 62 \%$ & 270 a 355 \\
Día 2 & $30,4 / 10,2$ & 158.000 & $3710 / 83 \%$ & 436 \\
Día 3 & $24,6 / 8,4$ & 76.000 & $7090 / 87 \%$ & 292 \\
\hline Hto: hematocrito. Hb: hemoglobina. PCR: proteína C reactiva. \\
\hline
\end{tabular}

Hto: hematocrito. Hb: hemoglobina. PCR: proteína C reactiva.
La instalación de un síndrome de secreción inadecuada de hormona antidiurética fue manejada con desmopresina, con respuesta regular. La monitorización farmacocinética de vancomicina comprobó concentraciones plasmáticas basales y pico que determinaron un área bajo la curva (AUC 24hrs / CIM=1 mg/L) de $423 \mathrm{mg} \mathrm{x} \mathrm{hr/L} \mathrm{donde} \mathrm{la}$ exposición del fármaco se interpretó como terapéuticamente efectiva para los modelos de PK/PD área bajo la curva dependiente. En ambos hemocultivos y en LCR se aisló S. pneumoniae con CIM por epsilometría (E-test) a penicilina $1,5 \mu \mathrm{g} / \mathrm{mL}$ y a cefotaxima $1,0 \mu \mathrm{g} / \mathrm{mL}$, y susceptible a cloranfenicol y vancomicina. Considerando la susceptibilidad in vitro de la cepa a cefotaxima, referida a sangre, se suspendió vancomicina luego de $48 \mathrm{~h}$ de haberse iniciado. Una nueva serie de dos hemocultivos de control a las $48 \mathrm{~h}$ de ingresar el paciente fue informada como negativa. El comportamiento de sus parámetros de laboratorio se resume en la Tabla 1.

El paciente tuvo una evolución tórpida, con marcado compromiso neurológico, ausencia de reflejo corneal, oculo-cefálico y oculo-palpebral, pupilas midriáticas, sin respuesta a estímulos luminosos ni dolorosos. Una nueva TAC efectuada a $24 \mathrm{~h}$ de su ingreso evidenció un extenso edema cerebral difuso, herniación amigdalina e hiperdensidad lineal de surcos cerebrales, con flujo doppler normal, lo que impidió la realización de una nueva punción lumbar. La monitorización electroencefalográfica mostró, a las $48 \mathrm{~h}$ de ingresado el paciente, escasa actividad difusa y una prueba de apnea que no gatilló ventilaciones. $\mathrm{Al} 6^{\circ}$ día de evolución se le realizó un nuevo EEG que objetivó la ausencia de actividad eléctrica, sin reactividad a estímulo sonoro ni doloroso, diagnosticándose muerte cerebral.

Se recibió y conoció post-mortem, el informe del laboratorio de referencia (Instituto de Salud Pública-ISP) que la cepa de $S$. pneumoniae aislada del LCR era resistente a penicilina (CIM $8 \mu \mathrm{g} / \mathrm{mL}$ ) y a cefotaxima (CIM $4 \mu \mathrm{g} / \mathrm{mL}$ ), por técnica de microdilución en caldo. Con posterioridad se confirmó desde el ISP la tipificación de S. pneumoniae serotipo 19 A.

Un estudio de anticuerpos antineumocóccicos comprobó concentraciones bajas $(<1,3 \mathrm{mg} / \mathrm{ml})$ para seis de los diez serotipos evaluados (Tabla 2). abundantes cocáceas grampositivas (Figura 1). Antes de transcurrido una hora desde su llegada a SU, se inició terapia antimicrobiana con ceftriaxona i.v. $100 \mathrm{mg} / \mathrm{kg} /$ día, en una dosis diaria, previa toma de hemocultivos. Ante la sospecha de etiología neumocóccica, se agregó al esquema anterior dexametasona i.v. 0,6 mg/ $\mathrm{kg} /$ día y tras 60 min desde el inicio de los corticoesteroides, vancomicina $60 \mathrm{mg} / \mathrm{kg} /$ día.

El paciente ingresó a UCI evolucionando grave, con necesidad de ventilación mecánica, compromiso hemodinámico, acidosis metabólica intensa, requiriendo apoyo vasoactivo con adrenalina y noradrenalina. Por la aparición de un síndrome convulsivo se agregó fenobarbital.

\section{Discusión}

Destaca en el caso presentado la gravedad y curso fatal de una ENI en un pre-escolar previamente sano y con vacunación anti-neumocóccica completa según el PNI de Chile. Como factores asociados a peor pronóstico y mayor letalidad, presentes al ingreso en pacientes con meningitis neumocóccica, han sido descritos la hipoglucorraquia $<20 \mathrm{mg} / \mathrm{dl}$, proteinorraquia $>250 \mathrm{mg} / \mathrm{dl}$, pleocitosis $<200 / \mathrm{mm}^{3}$, hemoglobina $<7 \mathrm{~g} / \mathrm{dl}$ y Glasgow $<10^{2}$. Cuatro 


\begin{tabular}{|c|c|}
\hline Serotipo & $\begin{array}{l}\text { Concentración plasmática }(\mathrm{mg} / \mathrm{mL}) \\
\text { (Rango normal }>1,3 \mathrm{mg} / \mathrm{mL})\end{array}$ \\
\hline Serotipo 1 (1) & 0,20 \\
\hline Serotipo 3 (3) & 0,55 \\
\hline Serotipo 4 (4) & 0,50 \\
\hline Serotipo 5 (5) & 1,50 \\
\hline Serotipo 26 (6B) & 1,85 \\
\hline Serotipo 68 (9V) & 0,55 \\
\hline Serotipo 14 (14) & 1,40 \\
\hline Serotipo 56 (18C) & 0,90 \\
\hline Serotipo 19 (19F) & 2,10 \\
\hline Serotipo 23 (23F) & 0,45 \\
\hline
\end{tabular}

de ellos se encontraban presentes en este paciente. $\mathrm{Su}$ comportamiento clínico, extremadamente grave, acompañado de una escasa respuesta inmune (leucopenia relativa en sangre y escasa celularidad en el LCR, en presencia de abundantes bacterias en la tinción de Gram directa) fueron motivos suficientes para sospechar de un defecto inmunológico, quizás una inmunodeficiencia específica para $S$. pneumoniae. Efectivamente, la medición de anticuerpos neumocóccicos comprobó que el paciente presentaba una respuesta deficiente a los antígenos contenidos en la vacuna 10-valente. La Food and Drug Administration (FDA) de E.U.A., estableció como valor que correlaciona con protección contra enfermedad invasora, una concentración plasmática de anticuerpos para cada serotipo de $1,3 \mathrm{mg} / \mathrm{ml}$, basado en estudios efectuados con la vacuna de polisacáridos. Con posterioridad se ha establecido que, para los individuos vacunados con preparados conjugados, una concentración plasmática de $0,35 \mathrm{mg} / \mathrm{ml}$ para cada serotipo predice protección contra una ENI, concepto que no es universalmente reconocido. En Chile, Lagos R. y cols. efectuaron un ensayo de inmunogenicidad en lactantes con la vacuna 10 -valente actualmente en uso en nuestro país, comprobando que sobre $95 \%$ de ellos alcanzó títulos post-vacunación sobre $1,3 \mathrm{mg} / \mathrm{ml}$ en 8 de los 10 serotipos y $87 \%$ lo lograron también con un $9^{\circ}$ serotipo $^{3}$. Nuestro paciente tenía concentraciones bajo $1,3 \mathrm{mg} / \mathrm{ml}$ en 6 de los 10 serotipos testeados, lo que sugiere la preexistencia en el niño de una incapacidad de responder ante los antígenos capsulares de $S$. pneumoniae. Al respecto, es entonces incierto si una vacunación del paciente con vacuna 13 -valente, que sí contiene antígeno del serotipo 19 A, hubiera evitado su enfermedad.

Los principales grupos con riesgo para desarrollar ENI son lactantes (niños bajo 2 años de edad), adultos sobre 65 años, pacientes con deficiencia de anticuerpos tipo IgG (incluida agammaglobulinemia y síndrome nefrótico), asplenia congénita o funcional, pacientes con enfermedad de células falciformes, aquellos con infección por $\mathrm{VIH}$, pacientes con implante coclear, fístula de LCR, hepatopatías crónicas, además de los usuarios de quimioterapia y corticoesteroides por tiempo prolongado $^{4}$.

En E.U.A., desde el año 2000 se inició la inmunización con vacuna conjugada heptavalente, que incluía a los principales serotipos causantes de ENI en el hemisferio norte. Las vacunas anti neumocóccicas conjugadas registradas posteriormente contenían antígenos capsulares de 10 y 13 serotipos $^{5}$. Además de su efecto protector directo sobre el individuo vacunado, las vacunas anti-neumocóccicas conjugadas han demostrado reducción de la portación nasofaríngea de los serotipos incluidos en las vacunas, induciendo inmunidad de rebaño en la población no vacunada. La cobertura otorgada por estas vacunas es variable dependiendo de los serotipos circulantes en cada región geográfica. Para los diferentes países del mundo que utilizan vacunas anti-neumocóccicas conjugadas se estima una disminución global de tasas de ENI por cualquier serotipo de alrededor de 50 a $60 \%$, con disminución de ENI por serotipos específicamente incluidos en las vacunas 10 y 13 valente, de 70 a 99\%, respectivamente ${ }^{6}$. Lagos R. y cols., evaluaron la cobertura teórica otorgada por estas vacunas para Chile, en base a un estudio de vigilancia activa de ENI efectuado en la Región Metropolitana entre los años 2000 y 2007, en lactantes y niños hospitalizados con edad igual o menor a 59 meses. Estos autores comprobaron que $54,4 \%$ de los casos de ENI correspondían a serotipos cubiertos por la vacuna 7 -valente, en tanto que $72,4 \%$ por la vacuna 10 -valente y finalmente $82,5 \%$ por la 13 -valente ${ }^{7}$.

Según información emanada del ISP, durante el año 2014, 65,2\% de los casos confirmados de ENI en lactantes corresponden a serotipos incluidos en la vacuna 10 -valente y $81,4 \%$ corresponden a serotipos incluidos en la vacuna 13 -valente.

En aquellos países que han incluido la vacunación anti-neumocóccica en sus programas de inmunización, se ha observado la aparición del llamado "fenómeno de reemplazo", correspondiente a la aparición de ENI causadas por serotipos no incluidos en la vacuna en una determinada región geográfica. Cuando se ha intentado cuantificar este fenómeno en estudios aleatorios/doble ciego, los datos no son concluyentes ${ }^{6,8}$.

En nuestro país, desde enero del año 2011 se introdujo al PNI la vacuna anti-neumocóccica 10 -valente (que incluye los antígenos 1, 4, 5, 6 B, 9 V, 14, 18 C, 19 F y 23 F), para todos los niños nacidos desde el 1 de noviembre de 2010, en esquema $2+1$ (inicialmente fue de $3+1$ ). Este esquema es avalado por la OMS y por el Grupo de Expertos en Asesoramiento Estratégico sobre Inmunización 
(SAGE) quienes recomiendan para aquellos países que decidan reducir costos en la incorporación de esta vacuna, el esquema $2+1$ con refuerzo entre los 9 y 15 meses de edad, destacando que el booster en un esquema $2+1$ induce una mayor respuesta que el esquema $3+0^{9}$.Según datos aportados por el ISP, fruto de una vigilancia pasiva de laboratorio sobre procesos invasores, para el período entre enero de 2007 y mayo de 2015 se confirmaron 6.398 casos de ENI. Al analizar los datos según grupo etario, las muestras correspondían, en orden decreciente, a adultos sobre 65 años de edad (20\%), adultos de 30-49 años $(13,9 \%)$ y niños bajo 12 meses de edad $(11,7 \%)$. Para este último grupo se observó una disminución de $66,2 \%$ en la confirmación de ENI entre 2007-2014, fenómeno observado además en el grupo de 12 a 23 meses, en que la confirmación de ENI, medida de esta forma, disminuyó en $64,4 \%$ en el mismo período ${ }^{1}$.

Cuando se analiza el número y porcentaje de casos de ENI por serotipo en lactantes bajo 12 meses de edad, se aprecia una disminución de la proporción de casos de los serotipos 1, 5 y 14 entre 2010 y 2014. Por otra parte, se ha observado un aumento relativo de serotipos no vaccinales como el serotipo 3 que elevó su frecuencia relativa desde $6 \%$ en el año 2010 a $13 \%$ en el $2013^{1}$. Éste es un serotipo inmunológicamente complejo; se postula que su cápsula es capaz de evadir al sistema inmune. En países con vacuna conjugada 13 -valente, que incluye el serotipo 3, se ha visto una protección sub-óptima para este serotipo ${ }^{10}$. Estudios epidemiológicos realizados en algunos estados, como Calgary y Alaska ${ }^{11,12}$, que han incorporado esquema de vacunación con PCV-13, han demostrado una disminución en la portación nasofaríngea reduciendo de $19,9 \%$ a $13,3 \%$. Respecto a los serotipos específicos, datos aportados por un estudio realizado en Milán ${ }^{13}$, demuestran que la reducción en la portación ha sido más significativa para el serotipo 19 A.

En relación al serotipo 19 A, éste también ha experimentado un alza relativa en el tiempo, desde $6 \%$ del total de casos de ENI en lactantes bajo 12 meses de edad, para el período 2007-2010 a 23\% durante el año 2014; para el grupo entre 12 a 23 meses aumenta de 6 a $27 \%$ y para el grupo de 24 a 59 meses lo hace de 6 a $32 \%$ para mismos períodos. Además se observó una menor susceptibilidad in vitro a penicilina y cefotaxima en casos de meningitis ${ }^{1}$. Este serotipo, según lo descrito en la literatura científica, se asocia a resistencia antimicrobiana y a gravedad clínica. Un estudio retrospectivo, realizado en E.U.A., comprobó que $S$. pneumoniae del serotipo 19 A surgió como uno de los serotipos más frecuentes luego de la incorporación de la vacuna heptavalente en dicho país, asociándose a cuadros clínicos graves, aumento de la frecuencia de mastoiditis y alta tasa de resistencia antimicrobiana. El mismo estudio analizó todas las ENI por serotipo 19 A en E.U.A. desde 1993 a 2011, donde se precisó su genotipo, así como también los patrones de resistencia. Se recolectó un total de 594 cepas de $S$. pneumoniae serotipo 19 A provenientes de cuadros invasores o bacteriémicos, principalmente neumonía, bacteriemia oculta, mastoiditis, meningitis e infecciones osteo-articulares. En el análisis realizado destacó un cambio en los genotipos prevalentes en el período 1993-2007 a lo observado con posterioridad al año 2007 , lo que refleja probablemente la presión del uso de antimicrobianos sumado al fenómeno post vacuna PCV- $7^{14}$. Otros estudios han mostrado un cambio en los patrones de resistencia antimicrobiana del serotipo $19 \mathrm{~A}$, incluso antes de la introducción de PCV-7, lo cual probablemente esté representando sólo el mecanismo de presión selectiva del uso de antimicrobianos ${ }^{15}$. Un ejemplo de ello queda demostrado en un estudio realizado en Canadá, donde a partir de los datos clínicos y microbiológicos de registros de ENI, se evidenció una mayor asociación de serotipo 19 A con meningitis en niños bajo 5 años de edad y una tasa de resistencia a múltiples antimicrobianos para el serotipo 19 A cercana a $20 \%$, en tanto que para otros serotipos esta tasa era menor a $1 \%{ }^{16}$.

Se ha recomendado efectuar estudio inmunológico en niños que experimentan una ENI, en especial si están debida y correctamente vacunados. Sobre esta materia, una revisión reciente fue realizada en Francia a una cohorte prospectiva de 163 niños con ENI, hospitalizados entre 2005 y 2011, con una edad media de 13 meses y una relación de mujeres versus hombres de 3:1. Tenían meningitis $87 \%$ de los casos y 17 niños padecieron de ENI recurrente. En ese país, se incorporó la vacuna 7-valente al PNI desde el año 2006. Su evaluación inmunológica sistemática incluyó hemograma, medición de inmunoglobulinas, concentraciones de complemento, citoquinas pro-inflamatorias y ecotomografía abdominal. El estudio inmunológico demostró alteración en 26 niños (16\%), de los cuales 17 presentaron inmunodeficiencia primaria, siendo la edad mayor de 2 años un factor independiente de mayor riesgo ( 26 vs $3 \% \mathrm{p}<0,001)$. Dentro de las alteraciones encontradas destacaban: deficiencia de fracción C2 o C3 del complemento, asplenia congénita aislada y agammaglobulinemia ligada al cromosoma X; además se detectó un déficit de anticuerpos anti-neumocóccicos en cuatro pacientes. A partir de esta revisión se concluyó la importancia de realizar una evaluación inmunológica en pacientes con ENI, en particular a los niños sobre 2 años, y así evaluar la necesidad de quimio-profilaxis y de refuerzo en vacunación, entre otras medidas ${ }^{17}$.

En síntesis, los factores probables determinantes de la enfermedad, gravedad y desenlace en nuestro paciente podrían ser explicados, en primer lugar por haber recibido una vacuna sin cobertura para el serotipo $19 \mathrm{~A}$, que fue el causante de esta infección. Un segundo factor probablemente determinante fue constatar una mala respuesta a la vacuna 10 -valente (baja concentración de anticuerpos 
anti-neumocóccicos), lo que sugiere una mala capacidad de respuesta inmune específica contra $S$. pneumoniae. Al enfermar, su respuesta inmunológica fue también pobre, evidenciada en la baja celularidad en el LCR en contraste con una presencia masiva de bacterias en la tinción de Gram directa del LCR. Un tercer factor a considerar fue la tardanza en la consulta ( $72 \mathrm{~h})$, lo que se tradujo en un diagnóstico e inicio de tratamiento tardíos. Finalmente, un cuarto factor que pudo haber influido en su desenlace fue la suspensión de vancomicina a las $48 \mathrm{~h}$ de haberse iniciado, por la interpretación errónea en el laboratorio de microbiología de la cepa causante de su enfermedad como sensible a cefalosporinas de tercera generación, considerando su patrón de susceptibilidad referido a sangre y no a LCR. Aún más, el informe a posteriori del ISP indicó que la cepa era aún más altamente resistente a $\beta$-lactámicos. Si esto incidió en el desenlace fatal del paciente es discutible; su gravedad fue extrema desde el ingreso al hospital sospechándose la muerte cerebral ya a los dos días de permanecer en la UCI, aun cuando los hemocultivos de control se negativizaron prontamente. No se tuvo control de la esterilización del LCR por las razones antes dadas.

En consecuencia, es necesario realizar una vigilancia activa de las ENI en nuestro país, para medir el impacto de la incorporación de la vacuna al PNI en los diferentes grupos etarios, evaluar un posible cambio a vacuna 13 -valente, establecer si los cambios de serotipos circulantes presentan un fenómeno similar a lo experimentado por otros países, así como evaluar la eventual asociación de presentación clínica y evolución según serotipo y tasas de resistencia antimicrobiana, para determinar conductas terapéuticas en la práctica clínica.

\section{Resumen}

La enfermedad neumocóccica invasora (ENI) sigue siendo una causa importante de morbilidad en el mundo y en nuestro país, si bien en Chile la incidencia ha disminuido luego de la incorporación de la vacuna neumocóccica conjugada 10-valente al Programa Nacional de Inmunizaciones (PNI). Uno de los efectos esperables luego de la vacunación programática con la vacuna antineumocóccica 10 -valente es el probable fenómeno de reemplazo, que corresponde a la presencia de ENI por serotipos no incluidos en la vacuna. En este contexto, se presenta el caso de un pre-escolar con meningitis neumocóccica causada por el serotipo $19 \mathrm{~A}$, de curso fatal. La presencia de casos de ENI en una etapa posterior a la implementación de la vacuna anti-neumocóccica en el PNI de Chile, demuestra la importancia de realizar una vigilancia activa, con el objetivo de conocer en forma detallada el impacto de la vacunación, la distribución de los serotipos circulantes y su correlación con los diferentes cuadros clínicos y su evolución.

\section{Referencias bibliográficas}

1.- Vigilancia de Enfermedad Invasora por Streptococcus pneumoniae. Chile, 2007 2015. Boletín Instituto de Salud Pública de Chile, 2015; 5, No 7 http://www.ispch.cl/sites/ default $/$ files/Bolet $\%$ C3\%ADn $\% 20 \mathrm{de} \% 20$ Vigilancia\%20de\%20Laboratorio\%20de \%20 Streptococcus\%20pneumoniae.pdf (Fecha de acceso: 6 de noviembre de 2015).

2.- Lovera D, Aranda C, Duarte M, Apodaca S, Acuña J, Arbo A. Predicción de la mortalidad de la meningitis neumocóccica en niños. Pediatr (Asunción) 2011; 38: 111- 7 .

3.- Lagos R, Muñoz A, Levine M, Lepetic A, Francois N, Yarzabal J, et al. Safety and immunogenicity of the 10-valent pneumococcal nontypable Haemophilus influenzae protein D conjugate vaccine (PHiD-CV) in Chilean children. Hum Vaccin 2011; 7: 511-22.

4.- Randle E, Ninis N, Inwald D. Invasive pneumococcal disease. Arch Dis Child Educ Pract Ed 2011; 96:183-90.

5.- $\quad$ Tan T Q. Pediatric invasive pneumococcal disease in the United States in the era of pneumococcal conjugate vaccines. Clin Microbiol Rev 2012; 25: 409-19.

6.- Weinberger D M, Malley R, Lipsitch M. Serotype replacement in disease following pneumococcal vaccination: A discussion of the evidence. Lancet 2011; 378: 1962-73.

7.- Lagos R, Muñoz A, San Martín O, Maldonado A, Hormázabal J C, Blackwelder W C, et al. Age-and serotypespecific pediatric invasive pneumococcal disease: insights from systematic survellance in Santiago, Chile, 1994-2007. J Infect Dis 2008; 198: 1809-17.

8.- Feikin D R, Kagucia E W, Loo J D, Link-Gelles R, Puhan M A, Cherian T, et al. Serotypes-specific changes in invasive pneumococcal disease after pneumococcal conjugate vaccine introduction: a pooled analysis of multiple surveillance sites. PLoS Med 2013; 10: e1001517.

9.- World Health Organization. Pneumococcal vaccines WHO position paper-2012. Weekly Epidemiol Record 2012; 87, 129-44.
Disponible en: http://www.who.int/wer/2012/ wer8714.pdf (Consultado el 6 de noviembre de 2015).

10.- Harboe Z B, Dalby T, Weinberger D M, Benfield T, Mølbak K, Slotved H C, et al. Impact of 13-valent pneumococcal conjugate vaccination in invasive pneumococcal disease incidence and mortality. Clin Infect Dis 2014; 59: 1066-73.

11.- Ricketson L J, Wood M L, Vanderkooi O G, MacDonald J C, Martin I E, Demczuk W H, et al. Trends in asymptomatic nasopharyngeal colonization with Streptococcus pneumoniae after introduction of the 13-valent pneumococcal conjugate vaccine in Calgary, Canada. Pediatr Infect Dis J 2014; 33: 724-30.

12.- Gounder P P, Bruce M G, Bruden D J, Singleton R J, Rudolph K, Hurlburt D A, et al. Alaska effect of the 13 valent pneumococcal conjugate vaccine on nasopharyngeal colonization by Streptococcus pneumoniae Alaska 2008-2012. J Infect Dis 2014; 209: 1251-8. 
13.- Zuccotti G, Mameli C, Daprai L, Garlaschib M L, Dilillo D, Bedogni G, et al. Serotype distribution and antimicrobial susceptibilities of nasopharyngeal isolates of Streptococcus pneumoniae from healthy children in the 13-valent pneumococcal conjugate vaccine era. Vaccine 2014; 32 : 527-34.

14.- Hulten K G, Kaplan S L, Lamberth L, Barson W J, Romero J R, Lin P L, et al.
Changes in Streptococcus pneumoniae serotype 19A invasive infection in children from 1993 to 2011. J Clin Microbiol 2013; 51: 1294-7.

15.- Choi E H, Kim S H, Eun B W, Kim S J, Kim N H, Lee J, et al. Streptococcus pneumoniae serotype 19A in children, South Korea. Emerg Infect Dis 2008; 14: 275-81.

16.- Ricketson L J, Vanderkooi O G, Wood M L,
Leal J, Kellner J D. Clinical features and outcomes of serotype 19A invasive pneumococcal disease in Calgary, Alberta. Can J Infect Dis Med Microbiol 2014; 25: e71-5.

17.- Gaschignard J, Levy C, Chrabieh M, Boisson B, Bost-Bru C, Dauger S, et al. Invasive pneumococcal disease in children can reveal a primary immunodeficiency. Clin Infect Dis 2014; 59: 244-51. 